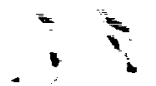

$$
\begin{aligned}
& N-39 \\
& 8204
\end{aligned}
$$

NASA Technical Memorandum 104047

\title{
PASSIVE DYNAMIC CONTROLLERS FOR NONLINEAR MECHANICAL SYSTEMS
}

Jer-Nan Juang, Shih-Chin Wu, Minh Phan, and Richard W. Longman

March 1991

\section{NNS凡}

National Aeronautics and

Space Administration

Langley Research Center

Hampton, Virginia 23665

(NASA-TM-104047) PASSIVE DYNAMIC CONTROLLERS FOR NONLINEAR MFCHANICAL SYSTEMS 


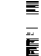




\title{
PASSIVE DYNAMIC CONTROLLERS FOR NON-LINEAR MECHANICAL SYSTEMS
}

\author{
Jer-Nan Juang, ${ }^{*}$ Shih-Chin $\mathrm{Wu}_{0}{ }^{+}$Minh Phan, ${ }^{* *}$ and Richard W. Longman ${ }^{++}$ \\ NASA Langley Research Center, Hampton, Virginia 23665
}

\begin{abstract}
A methodology for model-independent controller design for controlling large angular motion of multi-body dynamic systems is outlined. The controlled system may consist of rigid and flexible components that undergo large rigid body motion and small elastic deformations. Control forces/torques are applied to drive the system, and at the same time suppress the vibrations due to flexibility of the components. The proposcd controller consists of passive second-order systems which may be designed with little knowledge of the system parameters, even if the controlled system is non-linear. Under rather general assumptions, the passive design assures that the closed loop system has guaranteed stability properties. Unlike positive real controller design, stabilization can be accomplished without direct velocity feedback. In addition, the second-order passive design allows dynamic fecdback controllers with considerable frecdom to tune for desired system response, and to avoid actuator saturation. After developing the basic mathematical formulation of the design methodology, simulation results are presented to illustrate the proposed approach applied to a flexible sixdegrec-of-freedom manipulator.
\end{abstract}

\section{INTRODUCTION}

In this paper, a controller design methodology is outlined for non-linear dynamic systems based on simulating the force/torque histories that would be applied by a chosen virtual system of masses, springs and dashpots. In a companion paper by Juang and Phan ${ }^{1}$, the corresponding theory for linear dynamic systems is presented. Here, nonlinear problems are addressed by generalizing the standard Liapunov stability theory to allow less restrictive conditions on the Liapunov function. A major advantage of the control approach is its ability to guarantee stability of the controlled system with very little knowledge of the system being controlled. It has the advantages of positive real controller design methods, but in addition it allows

\footnotetext{
* Principal Scientist, Fellow, AIAA.

+ Engineer, Lockheed Engineering and Sciences Company.

** National Rescarch Council Rescarch Associate.

${ }^{++}$National Research Council Senior Rescarch

Associale. Also, Professor of Mochanical

Engincering, Columbia University, Fellow, AIAA.
}

use of more realistic choices for sensors. It allows dynamic controllers, and it easily handles a nonlinear environment. A secondary advantage is the physical intuition available to help guide the control system design. Both regulator (or vibration suppression) problems, and end-point control (or slewing) problems can be treated. The approach taken here exploits the work-energy rate principle in designing controllers as suggested by $O h$, Vadali and Junkins ${ }^{2}$.

The controller design methods developed here rely on the guarantecd energy dissipation produced by a virtual massspring-dashpot system for their stability properties. In the large flexible spacecraft shape control problem, Canavin ${ }^{3}$ was one of the first proponents of collocated velocity sensors and force actuators with pure velocity feedback. Such a control approach has the same guaranteed stability properties as the proposed design method, but the proposed method is more general, and does not necessarily require velocity feedback. Hyperstability, and positive real controller design methods are other closely related bodies of knowledge, Popov ${ }^{4-5}$. A positive real dynamic system is one which can be realized by purely passive electrical elements, Newcomb ${ }^{6}$. Benhabib, Iwens, and Jackson ${ }^{7}$ were the first to suggest applying such concepts from network theory for shape control of large flexible spacecraft. Flashner ${ }^{8}$ considers applications to robotics. The design of linear quadratic regulator controllers that are constraincd to be positive real is studied in Sevaston and Longman. ${ }^{9}$

The proposed approach is much more general than typical positive real controller design. For shape control of large nexible spacecraft, positive real theory requires the use of collocated velocity sensors and force actuators (or angular rate sensors and torque actuators). The requirement of velocity sensors is very restrictive in hardware implementation. Here the same type of stability robustness properties are obtained as in positive real theory, but the use of position, acceleration, and velocity sensors is allowed, either alone or in combinations. Collocation of sensors and actuators is still required. The controller design problem is formulated in terms of a virtual mechanical system which supplies considerable intuition to guide the control system design step.

This paper starts with a brief review of passive controller design for lincar systems as developed in Ref. 1. The basic results for the linear case are then generalized to the 
non-linear case. Insight into the passive control laws is given in the section entitled Physical Interpretation. A generic configuration similar to the Shuttle's Remote Manipulator System (RMS) is used as an example to illustrate the proposed controller design approach.

\section{BASIC RESULTS FOR LINEAR SYSTEMS}

The virtual spring-mass-damper approach to passive controller design is first described for linear dynamic systems as in Ref. 1. Extension of the theory to general non-linear multiple body dynamic systems is then made.

A general non-gyroscopic linear dynamic system can be represented as a system of second-order constant coefficient ordinary differential equations

$$
\begin{gathered}
M \ddot{x}+D \dot{x}+K x=B u \\
y=H_{a} \ddot{x}+H_{v} \dot{x}+H_{d} x
\end{gathered}
$$

where $x$ is an $n \times 1$ coordinate vector, and $M, D$, and $K$ are the symmetric mass, damping and stiffness matrices, respectively. The $n \times p$ influence matrix $B$ describes the actuator force distributions for the $p \times I$ control force vector $u$ (or generalized force). Typically, matrix $M$ is positive definite whereas $D$ and $K$ are positive definite or positive semi-definite. In the absence of rigid-body motion, $K$ is positive definite. Equation (2) is a measurement equation having $y$ as the $m \times I$ measurement vector, $H_{a}, H_{v}$, and $H_{d}$ are the $m \times n$ acceleration, velocity, and displacement influence matrices, respectively.

Assume that the controller to be designed has a set of second-order dynamic equations and measurement equations similar to the system equations in (1) and (2) above

$$
\begin{aligned}
& M_{c} \ddot{x}_{c}+D_{c} \dot{x}_{c}+K_{c} x_{c}=B_{c} u_{c} \\
& y_{c}=H_{a c} \ddot{x}_{c}+H_{v c} \dot{x}_{c}+H_{d c} x_{c}
\end{aligned}
$$

Here $x_{c}$ is the controller state vector of dimension $n_{C}$, and $M_{C}, D_{C}$, and $K_{C}$ are thought of as the controller mass, damping, and stiffness matrices, respectively. These matrices are chosen to be symmetric and positive definite to make the controller equations asymptotically stable. The $n_{C} \times m$ influence matrix $B_{C}$ describes the force distributions for the $m \times l$ input force vector $u_{c}$. Equation (4) is the controller measurement equation having $y_{C}$ as the measurcment vector of length $p, H_{a c}$ the $p \times n_{C}$ acceleration influence matrix, $H_{v c}$ the $p \times n_{c}$ velocity influence matrix and $H_{d c}$ the $p \times n_{c}$ displacement influence matrix. The quantities $M_{c}, D_{c}, K_{c}, B_{c}, H_{a c}$, $H_{d c}$, and $H_{v c}$ are the design parameters for the controller. Let the dynamic system in (1) and (2) be connected to the controller systcm in (3) and (4) in such a way that the output of the controller is the input to the dynamic system, and the output of the dynamic system is the input to the controller, i.e.,

$$
\begin{gathered}
u=y_{c}=H_{a c} \ddot{x}_{c}+H_{v c} \dot{x}_{c}+H_{d c} x_{c} \\
u_{c}=y=H_{a} \ddot{x}+H_{v} \dot{x}+H_{d} x
\end{gathered}
$$

The overall closed-loop system then becomes

where

$$
M_{1} \ddot{x}_{t}+D_{1} \dot{x}_{t}+K_{1} x_{1}=0
$$

$$
\begin{aligned}
M_{\imath} & =\left[\begin{array}{cc}
M & -B H_{\alpha c} \\
-B_{c} H_{a} & M_{c}
\end{array}\right], D_{\imath}=\left[\begin{array}{cc}
D & -B H_{v c} \\
-B_{c} H_{v} & D_{c}
\end{array}\right], \\
K_{\iota} & =\left[\begin{array}{cc}
K & -B H_{d c} \\
-B_{c} H_{d} & K_{c}
\end{array}\right], x_{t}=\left[\begin{array}{l}
x \\
x_{c}
\end{array}\right]
\end{aligned}
$$

If the system parameters, $M, D, K, H_{a}, H_{d}$, and $H_{\nu}$ are known, then the controller parameters, $M_{C}, D_{c}, K_{C}, H_{a c}$, $H_{d c}$, and $H_{v c}$ can be designed such that the closed-loop system matrices, $M_{t}, D_{t}$, and $K_{t}$ are symmetric and positive definite. This makes the closed-loop system, Eq. (7), asymptotically stable. However, it is of interest to design controllers that are insensitive to the system parameters. In the following development, this is indeed possible provided a certain modification to the control equation is made, and a certain condition on the actuator and sensor placement is satisfied. First, note the similarity in structures in the closed-loop system matrices $M_{t}, D_{t}$, and $K_{t}$. This means that the basic design procedure for the controller parameters that appear in each of the matrices above is the same. In particular, the case for designing the parameters in the stiffness matrix $K_{l}$ is illustrated here. Let the actuators be located in such a way that the control influence matrix of the system equation, $B$, can be expressed by

$$
B^{T}=Q_{b} H_{d}
$$

then if the control influence matrix of the control equation, $B_{C}$, is designed such that

$$
Q_{b}^{T} H_{d c}=B_{c}^{T}
$$

for any given matrix $H_{d c}$, then the resulting closed-loop stiffness matrix $K_{t}$ is symmetric. To derive the conditions that would make $K_{t}$ positive definite, it is adequate to consider the special case where $H_{a}=H_{a c}=H_{v}=H_{v c}=0$. Furthermore, let the input force in (5) be modified as

$$
u=y_{c}-G y=H_{d c} x_{c}-G H_{d} x
$$

where $G$ is a gain matrix to be determined. Also, let

$$
B_{c}=K_{c} \bar{B}_{c} \text { or } \bar{B}_{c}=K_{c}^{-1} B_{c}
$$

and the gain matrix $G$ be 


$$
G=H_{d c} \bar{B}_{c}
$$

then it can be shown that the closed-loop stiffness matrix in this case becomes

$$
K_{1}=\left[\begin{array}{cc}
K+H_{d}^{T} \bar{B}_{c}^{T} K_{c} \bar{B}_{c} H_{d} & -H_{d}^{T} \bar{B}_{c}^{T} K_{c} \\
-K_{c} \bar{B}_{c} H_{d} & K_{c}
\end{array}\right]
$$

which, in addition to being symmetric, is positive definite if the system stiffness matrix $K$ is at least positive semidefinite. The design procedure for the other controller parameters in the closed-loop system mass and damping matrices are similar.

\section{GENERALIZATIONS TO MULTIPLE BODY DYNAMIC SYSTEMS}

The basic results for linear systems can be generalized to the non-linear case. This section outlines the overall methodology for extension to non-linear systems. A detailed development of all the possibilities involved is deferred to a later paper. Certain assumptions are made in the course of the development here which must be satisfied by the physical system being controlled. Various possible controller structures are considered for different types of sensor feedback, and then a Liapunov like approach is taken to address the stability question. First consider multiple rigid body systems. The case of multiple flexible body systems is discussed later. Also, mechanical systems are considered which have no external forces applied, such as robots in space.

\section{Velocity Feedback}

We start by considering controllers that use velocity feedback only. Since no position measurements are made, no attempt is made to control position. Such controllers are important by themselves for vibration suppression, and are also important here as one building block in more general controller designs discussed below. Let $T$ be the total kinctic energy of a mechanical system (linear or nonlinear) with $\mathrm{p}$ control actuators at $\mathrm{p}$ physical locations which are described by p generalized coordinates $x_{a i}, \mathrm{i}=$ $1,2, \ldots, p$. These generalized coordinates and their derivatives are measurable quantities such as displacements, velocitics, accelerations or their angular cquivalents. If the mechanical system is holonomic and scleronomic (no explicit time dependence), a basic result of analytical mechanics relates the time derivative of the total kinetic encrgy, $\dot{T}$, and the applicd forces as 2

$$
\frac{d T}{d t}=u^{T} \dot{x}_{a}
$$

where $u=\left(\begin{array}{llll}u_{1} & u_{2} & \cdots & u_{p}\end{array}\right)^{T}$ is the control vector with $u_{i}(i=1,2, \ldots, p)$ representing the generalized control force associated with the generalized coordinate $x_{a i}$, and $x_{a}=\left(\begin{array}{llll}x_{a 1} & x_{a 2} & \cdots & x_{a p}\end{array}\right)^{T}$ is a gencralized coordinate vector. The physical force or torque for each actuator is a function of these generalized forces through kinematic relations. Equation (14) is referred to as the work-energy rate principle prensented in Ref. 2 . It indicates that the rate of change of total kinetic energy is equal to the rate of change of work produced by applied forces.

Consider $T$ as a Liapunov function

$$
L=L\left(x_{a}, \dot{x}_{a}, \bar{x}, \dot{\bar{x}}\right)=T\left(x_{a}, \dot{x}_{a}, \bar{x}, \dot{\bar{x}}\right)
$$

where $\bar{x}$ denotes a vector of additional generalized coordinates which need not be measurable. It is assumed that there are no generalized forces generated by the controller for these extra coordinates. The time derivative of the Liapunov function, $\dot{L}$, becomes

$$
i=\frac{d L}{d l}=\frac{d T}{d t}=u^{T} \dot{x}_{a}
$$

Let the gencralized control vector $u$ be chosen such that

$$
u=-D \dot{x}_{a}
$$

Then it follows that

$$
\frac{d L}{d t}=-\dot{x}_{a}^{T} D \dot{x}_{a}
$$

which is negative semi-definite in $\dot{x}_{a}, \dot{\bar{x}}$ space when $\mathrm{D}$ in Eq. (18) is chosen as positive definite. Since the Liapunov function $L$ is positive definite in the variables $\dot{x}_{a}, \dot{\bar{x}}$, and its time rate of change $\dot{L}$ is only positive semi-definite in the same set of variables, some caution must be exercised with regard to a conclusion concerning the asymptotic stability of the overall system. If it is assumed that the mechanical structure of the system is such that $\dot{x}_{a} \equiv 0$ implies $\dot{\bar{x}} \equiv 0$, then it is not possible to have $\dot{L}$ identically zero, and yet have the kinetic energy $L$ fail to vanish identically. This assumption is satisfied provided that the manifold of the $\left(\dot{x}_{a}, \dot{\bar{x}}\right)$ space on which the time rate of change of the Liapunov function vanishes identically,

$$
\frac{d L}{d l}=-\dot{x}_{a}^{T} D \dot{x}_{a} \equiv 0
$$

does not contain any arc of a trajectory, and in this case one can conclude that the trivial solution

$$
\dot{x}_{a}=0, \quad \dot{\bar{x}}=0
$$

is asymptotically stable. It is possible, however, to allow the trajectorics to cross the manifold, as long as they do not remain in it except when at the origin. The time rate of change of the Liapunov function considered in 
this case is only negative semi-definite, but the fact that no physical trajectory arcs lie in the manifold insures that over any finite time interval encrgy is dissipated. For our control problem, the assumption has the physical implication that it is not possible to have some coordinates still in motion without disturbing any of the controlled coordinates. In practice, this condition may be accomplished by having a sufficient number of sensors collocated with the actuators at proper locations.

\section{Displacement Feedback}

Consider displacement feedback in order to control position, or altematively to suppress vibrational motion as in the above section but without velocity measurements being available. Choose the Liapunov function

$$
L=L\left(x_{a}, \dot{x}_{a}, x_{c}, \dot{x}_{c}, \bar{x}, \dot{\bar{x}}\right)
$$

where

$$
L=T+\frac{1}{2} \dot{x}_{c}^{T} M_{c} \dot{x}_{c}+\frac{1}{2}\left(x_{a}-x_{c}\right)^{T} K_{c_{1}}\left(x_{a}-x_{c}\right)+\frac{1}{2} x_{c}^{T} K_{c_{2}} x_{c}
$$

where $x_{c}=\left(\begin{array}{llll}x_{c 1} & x_{c 2} & \cdots & x_{c p}\end{array}\right)^{T}$ is the displacement vector corresponding to a control (virtual) mass matrix $M_{c}$, and $M_{c}, K_{c_{1}}$ and $K_{c_{2}}$ are arbitrary positive definitc matrices to be determined later. The time derivative of the Liapunov function, $i$, becomes

$$
\frac{d L}{d t}=u^{T} \dot{x}_{a}+\dot{x}_{c}^{T} M_{c} \ddot{x}_{c}+\left(\dot{x}_{a}-\dot{x}_{c}\right)^{T} K_{c_{1}}\left(x_{a}-x_{c}\right)+\dot{x}_{c}^{T} K_{c_{2}} x_{c}
$$

Let $\mathrm{u}$ be chosen such that

$$
u=-K_{c_{1}}\left(x_{a}-x_{c}\right)
$$

This is a model-independent feedback law which involves only the measurable generalized coordinate vector $x_{a}$ of the system and the computable displacement $x_{c}$ from a controller to be determined later. The positive definite matrix $K_{c_{1}}$ and the displacement vector $x_{c}$ are determined to make $i$ negative semi-definite. Substitution of Eq. (24) into Eq. (23) yields

$$
\begin{aligned}
\frac{d L}{d l} & =-\left(x_{a}-x_{c}\right)^{T} K_{c_{1}} \dot{x}_{a}+\dot{x}_{c}^{T} M_{c} \ddot{x}_{c} \\
& +\left(\dot{x}_{a}-\dot{x}_{c}\right)^{T} K_{c}\left(x_{a}-x_{c}\right)+\dot{x}_{c}^{T} K_{c_{2}} x_{c} \\
& =\dot{x}_{c}^{T} M_{c} \ddot{x}_{c}-\dot{x}_{c}^{T} K_{q}\left(x_{a}-x_{c}\right)+\dot{x}_{c}^{T} K_{c_{2}} x_{c} \\
& =\dot{x}_{c}^{T}\left[M_{c} \ddot{x}_{c}-K_{c_{1}}\left(x_{a}-x_{c}\right)+K_{c_{2}} x_{c}\right]
\end{aligned}
$$

Let the quantity in the square bracket be

$$
M_{c} \ddot{x}_{c}-K_{c_{1}}\left(x_{a}-x_{c}\right)+K_{c_{2}} x_{c}=-D_{c} \dot{x}_{c}
$$

or

$$
M_{c} \ddot{x}_{c}+D_{c} \dot{x}_{c}+\left(K_{c_{1}}+K_{c_{2}}\right) x_{c}=K_{q_{1}} x_{a}
$$

The right hand side of Eq. (27) involves only the mcasurable generalized coordinate vector $x_{a}$ which in tum determines the quantitics $x_{c}, \dot{x}_{c}$ by computation. Equation (27) is a lincar dynamic system represented by the controller mass matrix $M_{c}$, damping matrix $D_{c}$ and stiffness matrix $\left(K_{c_{1}}+K_{c_{2}}\right)$ which are all positive definitc. The generalized control vector $u$ is generated by simulating the force histories that would be applied by the chosen virtual (controller) mass-spring-dashpot system described by Eq. (27). Its physical interpretation will be shown later. Combination of Eqs. (25) and (26) yields

$$
\dot{L}=-\dot{x}_{c}^{T} D_{c} \dot{x}_{c}
$$

which is negative semi-definite in the variables $x_{a}, \dot{x}_{a}, x_{c}, \dot{x}_{c}, \bar{x}, \dot{\bar{x}}$. The objective of the control is to obtain

$$
\lim _{t \rightarrow \infty} x_{a}(t)=\lim _{t \rightarrow \infty} x_{c}(t)=0
$$

This can be accomplished provided the Liapunov function considered in Eq. (22) approaches zero asymptotically. Since $L$ is only negative semi-definite in the variables $x_{a}, \dot{x}_{a}, x_{c}, \dot{x}_{c}, \bar{x}, \dot{\bar{x}}$, in general it is possible to have $\dot{L}$ vanish, yet the Liapunov function L itself does not. Assuming that the system is configured such that it is not possible to have some coordinates still in motion without disturbing any of the controlled coordinates, i.e., $\dot{x}_{a} \equiv 0$ implies $\dot{\bar{x}} \equiv 0$, then in the limit L must approach zero as $t$ tends to infinity. This can be seen by considering the case where $\dot{x}_{c} \equiv 0$. Note that $\dot{x}_{c} \equiv 0$ implies $\dot{x}_{a} \equiv 0$. Thercfore, the first two terms in the Liapunov function, Eq. (22), vanish. Furthermore, in the absence of external forces other than control forces, assume that $\dot{x}_{c} \equiv 0$ implics $x_{c} \equiv 0$, which from Eq. (27) yields $x_{a} \equiv 0$ provided $K_{q_{1}}$ is invertible. This makes the last two terms in the Liapunov function vanish as well.

Computation of the quantity $x_{c}$ requires measurement of the vector $x_{a}$ as shown in Eq. (27). Therefore, when velocity sensors are not available, so that the control law cannot depend on measuring $\dot{x}_{a}$ as in the previous section, it is still possible to make $\dot{L}$ negative semidefinite using the feedback law given in Eq. (24), and accomplish vibration suppression The positive definite matrices $M_{c}, D_{c}, K_{q_{1}}$ and $K_{c_{2}}$ are arbitrary and can be chosen to meet performance requirements. 


\section{Displacement and Acceleration Feedback}

Feedback laws using acceleration measurements can be derived similarly. This is an important case for vibration suppression in structures since acceleration measurements are much more easily made than velocity measurements. Consider a Liapunov function of the form

$$
\begin{aligned}
L= & T+\frac{1}{2}\left(\dot{x}_{a}+\dot{x}_{c}\right)^{T} M_{c}\left(\dot{x}_{a}+\dot{x}_{c}\right)+\frac{1}{2} x_{c}^{T} K_{q} x_{c} \\
& +\frac{1}{2}\left(x_{a}+x_{c}\right)^{T} K_{c_{2}}\left(x_{a}+x_{c}\right)
\end{aligned}
$$

The time derivative of $\mathrm{L}$ then becomes

$$
\begin{aligned}
\frac{d L}{d t}= & u^{T} \dot{x}_{a}+\left(\dot{x}_{a}+\dot{x}_{c}\right)^{T} M_{c}\left(\ddot{x}_{a}+\ddot{x}_{c}\right)+\dot{x}_{c}^{T} K_{c_{1}} x_{c} \\
& +\left(\dot{x}_{a}+\dot{x}_{c}\right)^{T} K_{c_{2}}\left(x_{a}+x_{c}\right)
\end{aligned}
$$

Let the control input be chosen such that

$$
u=-M_{c}\left(\ddot{x}_{a}+\ddot{x}_{c}\right)-K_{c_{2}}\left(x_{a}+x_{c}\right)
$$

then the time rate of change of the Liapunov function becomes

$$
\begin{aligned}
\frac{d L}{d t}= & -\left(\ddot{x}_{a}+\ddot{x}_{c}\right)^{T} M_{c} \dot{x}_{a}-\left(x_{a}+x_{c}\right)^{T} K_{c_{2}} \dot{x}_{a} \\
& +\left(\dot{x}_{a}+\dot{x}_{c}\right)^{T} M_{c}\left(\ddot{x}_{a}+\ddot{x}_{c}\right)+x_{c}^{T} K_{c} \dot{x}_{c} \\
& +\left(\dot{x}_{a}+\dot{x}_{c}\right)^{T} K_{c_{2}}\left(x_{a}+x_{c}\right) \\
= & \dot{x}_{c}^{T} M_{c}\left(\ddot{x}_{a}+\ddot{x}_{c}\right)+x_{c}^{T} K_{c_{1}} \dot{x}_{c}+\dot{x}_{c}^{T} K_{c_{2}}\left(x_{a}+x_{c}\right) \\
& -\left(x_{a}+x_{c}\right)^{T} K_{c_{2}} \dot{x}_{a}+\sum_{i=1}^{n} \dot{x}_{a}^{T} K_{c_{2}}\left(x_{a}+x_{c}\right) \\
= & \dot{x}_{c}^{T}\left[K_{c_{1}} x_{c}+M_{c}\left(\ddot{x}_{a}+\ddot{x}_{c}\right)+K_{c_{2}}\left(x_{a}+x_{c}\right)\right]
\end{aligned}
$$

If the quantity in the square bracket in the above equation is set to be

$$
K_{c_{1}} x_{c}+M_{c}\left(\ddot{x}_{a}+\ddot{x}_{c}\right)+K_{c_{2}}\left(x_{a}+x_{c}\right)=-D_{c} \dot{x}_{c}
$$

or

$$
M_{c} \ddot{x}_{c}+D_{c} \dot{x}_{c}+\left(K_{c_{1}}+K_{c_{2}}\right) x_{c}=-M_{c} \ddot{x}_{a}-K_{c_{2}} x_{a}
$$

wherc $D_{c}$ is an arbitrary positive definite matrix, then Eq. (33) becomes

$$
\frac{d L}{d t}=-\dot{x}_{c}^{T} D_{c} \dot{x}_{c}
$$

which is negative semi-definite in the variables $x_{a}, \dot{x}_{a}, x_{c}, \dot{x}_{c}, \bar{x}, \dot{\bar{x}}$. Note that even in the absence of velocity measurements, the feedback control law given in Eq. (32) which is based on acceleration and displacement measurements is sufficient to make $\dot{L}$ negative semidefinite. Again, assuming that $\dot{x}_{a} \equiv 0$ implies $\dot{\bar{x}} \equiv 0$, it can be concluded that $L$ approaches zero as $t$ tends to infinity. The line of argument follows closely the previous development in the case of displacement feedback and hence is omitted.

\section{Displacement, Velocity, and Acceleration Feedback}

The general case of displacement, velocily, and acceleration feedback can be easily derived by considering a Liapunov function of the form

$$
L=T+L_{1}+L_{2}
$$

where

$$
\begin{aligned}
L_{1}= & \frac{1}{2} \dot{x}_{c d}^{T} M_{c d} \dot{x}_{c d} \\
& +\frac{1}{2}\left(x_{a}-x_{c d}\right)^{T} K_{c d_{1}}\left(x_{a}-x_{c d}\right)+\frac{1}{2} x_{c d}^{T} K_{c d_{2}} x_{c d} \\
L_{2}= & \frac{1}{2}\left(\dot{x}_{a}+\dot{x}_{c a}\right)^{T} M_{c a}\left(\dot{x}_{a}+\dot{x}_{c a}\right) \\
& +\frac{1}{2} x_{c a}^{T} K_{c a} x_{c a}+\frac{1}{2}\left(x_{a}+x_{c a}\right)^{T} K_{c a_{2}}\left(x_{a}+x_{c a}\right)
\end{aligned}
$$

The control law is then of the form

$$
\begin{aligned}
u= & -D \dot{x}_{a}-K_{c \alpha_{1}}\left(x_{a}-x_{c d}\right)-M_{c a}\left(\ddot{x}_{a}+\ddot{x}_{c a}\right) \\
& -K_{c a_{2}}\left(x_{a}+x_{c a}\right)
\end{aligned}
$$

where the vectors $x_{c d}$ and $x_{c a}$ are determined from

$$
\begin{aligned}
& M_{c d} \ddot{x}_{c d}+D_{c d} \dot{x}_{c d}+\left(K_{c d_{1}}+K_{c d_{2}}\right) x_{c d}=-K_{c d_{1}} x_{a} \\
& M_{c a} \ddot{x}_{c a}+D_{c a} \dot{x}_{c a}+\left(K_{c a_{1}}+K_{c a_{2}}\right) x_{c a}=-M_{c a} \ddot{x}_{a}-K_{c q_{2}} x_{a}
\end{aligned}
$$

The cocfficient matrices as well as $K_{c \alpha_{1}}$ and $K_{c a_{2}}$ in Eq. (39) are all required to be positive definite. The time rate of change of the Liapunov function then becomes

$$
\dot{L}=-\dot{x}_{a}^{T} D \dot{x}_{a}-\dot{x}_{c d}^{T} D_{c d} \dot{x}_{c d}-\dot{x}_{c a}^{T} D_{c a} \dot{x}_{c a}
$$

which is negative semi-definite. The proof of stability parallels the previous case.

\section{Generalization to Multiple Flexible Body Systems with Viscous Friction}

Including flexibility into the bodies of the system, introduces a potential energy of deformation. In many cases such as robots with controllers at each joint, this potential encrgy is special in the sense that it does not alter the equilibrium position of the system including the virtual controller. Since the potential energy function does not change the equilibrium position, or introduce new equilibria, there is no need to solve for the equilibrium before studying stability. To include such a potential 
energy in the analysis, one simply notes that the time rate of change of the kinetic energy in Eq. (14) becomes the time rate of change of the total energy 2 so that

$$
\frac{d}{d t}(T+V)=-2 F_{d}+u^{T} \dot{x}_{a}
$$

where $F_{d}$ is the quadratic Rayleigh's dissipation function included here to handle any viscous damping in the system. All the theory developed above applies again. One simply replaces the kinetic energy $T$ by the total mechanical energy $E=T+V$ of the system in the related expressions.

\section{PHYSICAL INTERPRETATION}

Insight into the control laws developed can be gained through the following physical interpretation in terms of mechanical analogs. It is these analogs that motivated the mathematical formulations above. For simplicity, consider only the single input and single output case. The matrices $M_{c}, D_{c}, K_{c}$ reduce to scalar quantities and are denoted by the lower case letuers $m_{c}, d_{c}, k_{c}$.

\section{Velocity Feedback}

The controller is given by Eq. (17). The control force applied is equivalent to the force transmitted to the system at the actuator location by a dashpot with a damping coefficient d. This is shown in Fig. 1.

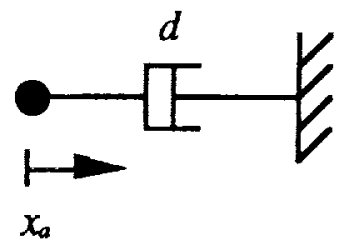

Figure 1: Velocity Feedback

If $x_{a}$ is an angular displacement, then the control law corresponds to an angular dashpot.

\section{Displacement Feedback}

The control law is given in Eq. (24). Examination of Eqs. (24) and (27) shows that the control force is equivalent to that provided by a mass-spring-dashpot system at the actuator location. This is shown in Fig. 2.

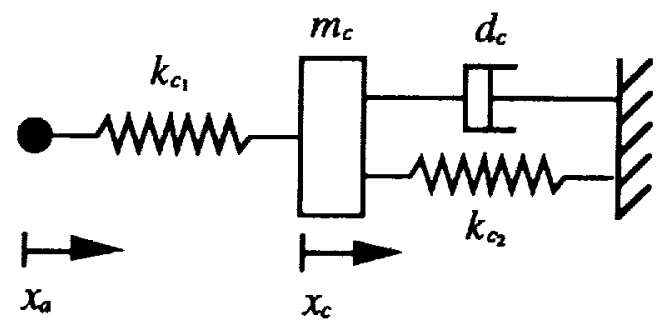

Figure 2: Displacement Feedback

In this case, the quantity $x_{a}$ is the measured inertial position of the system at the actuator location, whereas the quantity $x_{c}$ is defined as the inertial position of the virtual mass $m_{c}$. The springs are considered as ideal springs, and their unstretched lengths can be considered to be zero. The corresponding block diagram for this case is given in Fig. 3.

\section{Displacement and Acceleration Feedback}

The control law is given in Eqs. (32) and (35). Examination of the controller equations verifies that the control force is equivalent to a mass-spring-dashpot system at the actuator location, with one important distinguishing feature. The position of the controller (virtual) mass $x_{c}$ shown in Fig. 4 is now defined relative to the measured incrtial position $x_{a}$ of the system at the actuator location whcreas in the displacement feedback

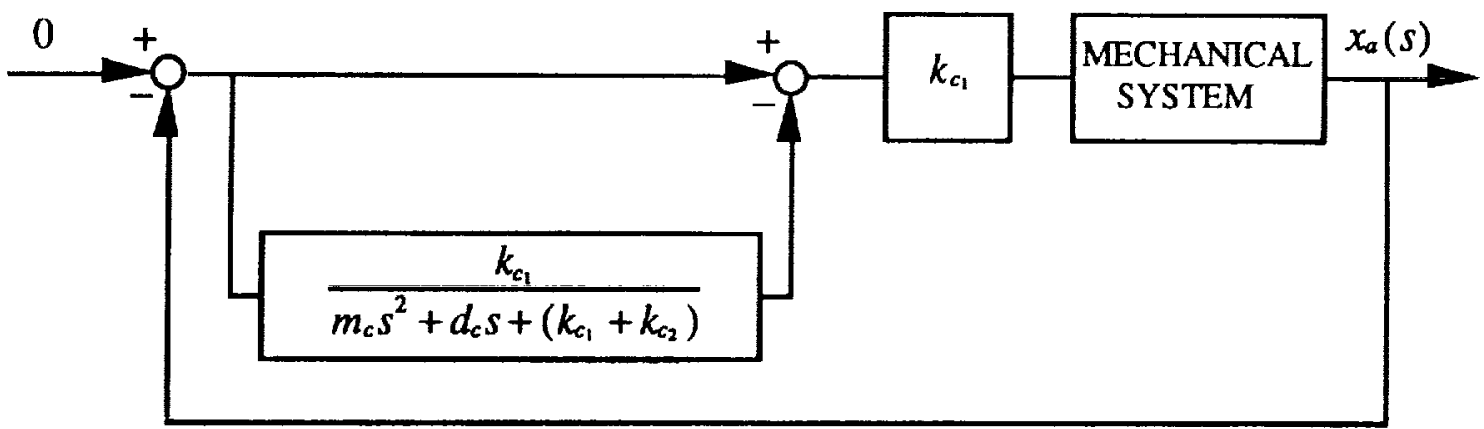

Figure 3: Displacement Feedback Controller 
case above, it is defined as an inertial position. The free lengths of the springs are taken as zero. The corresponding block diagram for this case is given in Fig. 5.

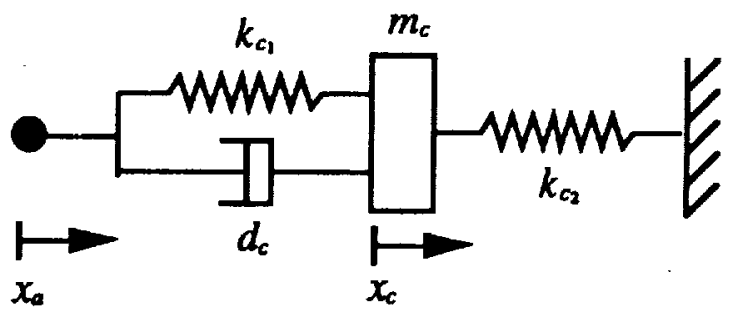

Figure 4: Displacement and Acceleration Feedback

Displacement, Velocity, and Acceleration Feedback

The control law is given in Eq. (38). The equivalent mass-spring-dashpot diagram for this case is given in Fig. 6 and the block diagram for this controller is given in Fig. 7.

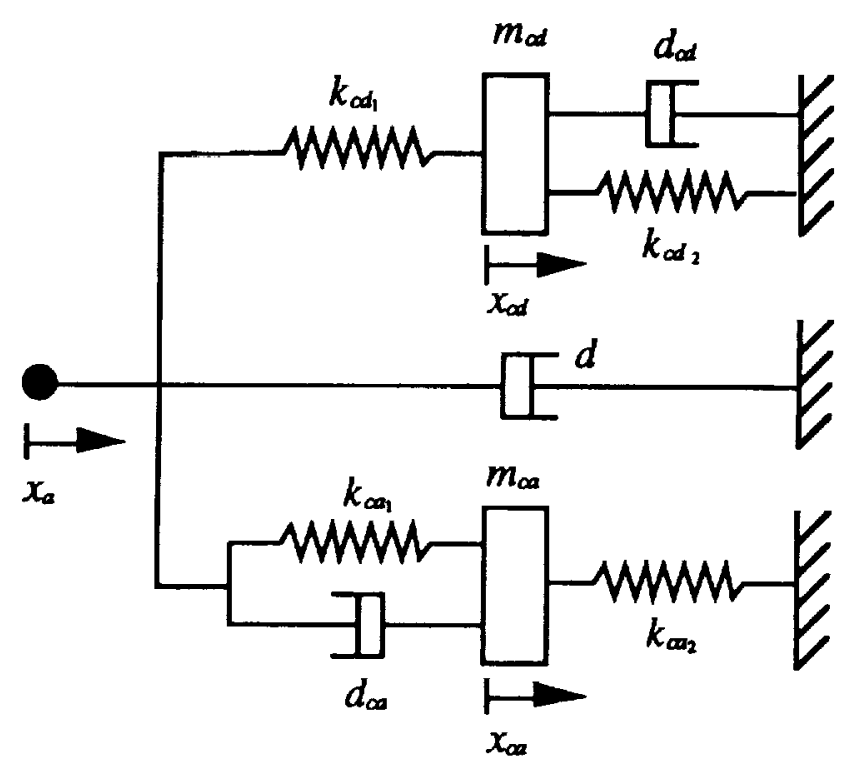

Figure 6: Displacement, Velocity, and Acceleration Feedback

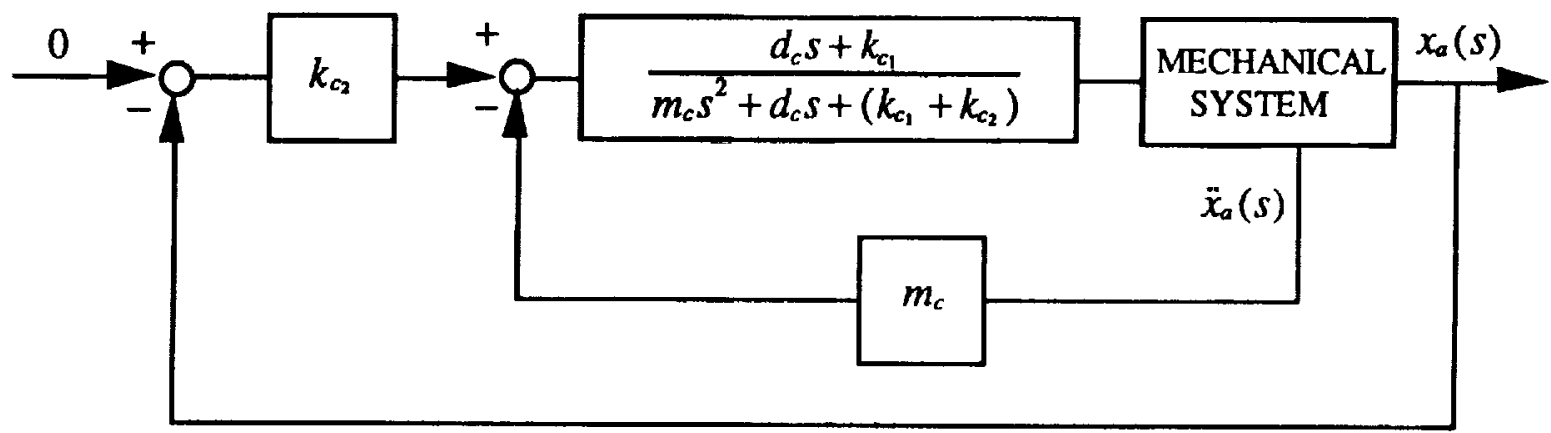

Figure 5: Displacement and Acceleration Feedback Controller

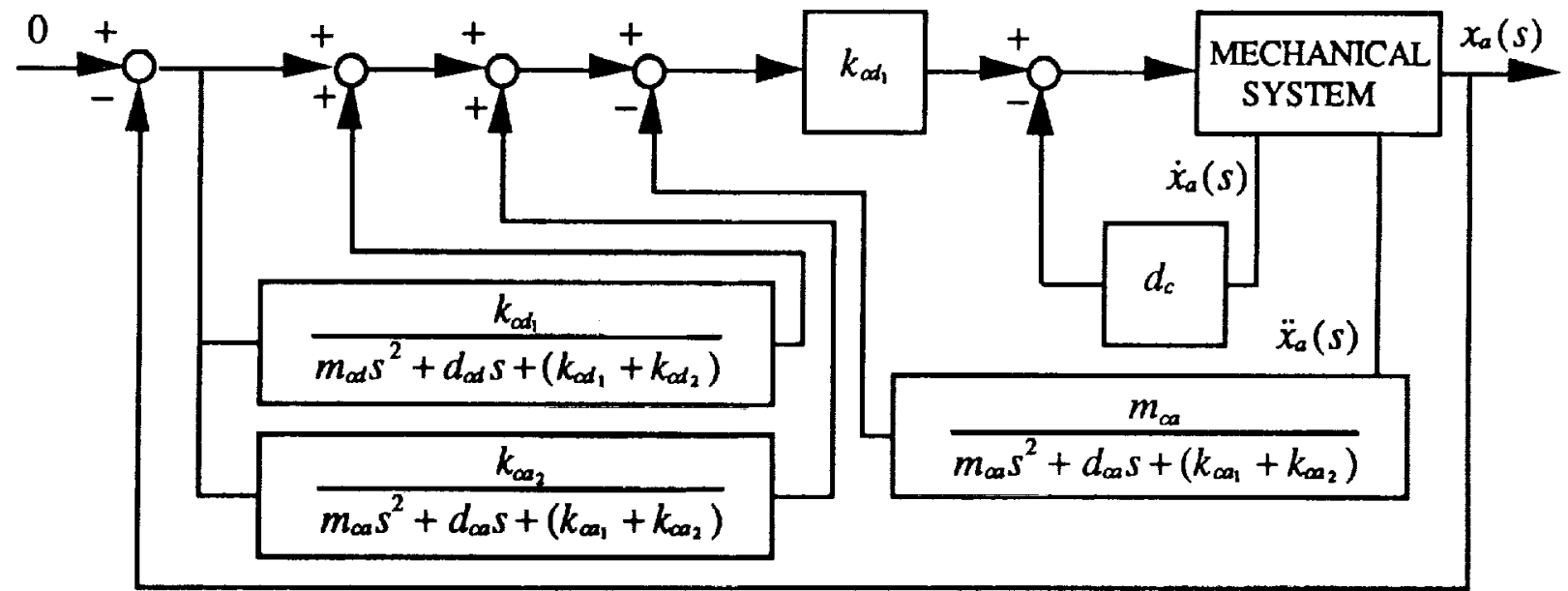

Figure 7: Displacement, Velocity, and Acceleration Feedback Controller 


\section{REMARKS}

Remark 1: Certain simplifications of the controllers developed here are worth noting. In both the displacement fcedback, and displacement and acceleration feedback cases, the inclusion of the spring characterized by its coefficient

$k_{c_{2}}$ is necessary for position control, but not vital for vibration suppression. This special feature allows one to design robust vibration suppression controllers with accelerometer feedback. In particular, setting $k_{c_{2}}=0$ in the displacement and acceleration feedback law, Eq.(32), results in a feedback law that is based on acceleration measurements alone. The corresponding diagram for this case is given in the Fig. 8.

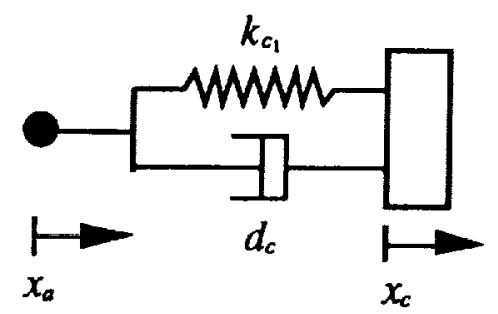

Figure 8: Acceleration Fcedback

Remark 2: In the absence of direct velocity measurements, it is possible to mimic direct velocity feedback by displacement measurements alone. Consider the case of displacement feedback as shown in Fig. 2. By

letling $k_{c_{2}}=0$, and choosing $k_{c_{1}}$ to be large, and $m_{c}$ to be small, the behavior of the resulting mass-springdashpot system approximates that of a single dashpot. This is equivalent to putting a controller zero near the origin but not at the origin.

Remark 3: It is also possible to approximate direct velocity feedback from displacement and acceleration

measurements. Refer to Fig. 4. If $k_{c_{1}}, m_{c}$ are chosen to be small, and $k_{c_{2}}$ to be large, then the behavior of the resultant mass-spring-dashpot system approximates that of a single dashpot. In the absence of displacement measurement $\left(k_{c_{2}}=0\right)$, the same effect can be achieved by choosing $k_{c_{1}}$ to be small, but $m_{c}$ to be large.

Remark 4: To implement the controller in Fig. 2 or 4, it is necessary to select the correct initial conditions for the controller coordinate $x_{c}$ to be used. There is a subtle difference between the vibration suppression problem and the end-point control problem by this approach, even though they may be viewed as the same conceptually. For vibration suppression, the controller consists of second-order dynamic systems whose equilibrium position of the controller mass is consistent with the desired equilibrium configuration of the structure. Assuming that the structure is initially at rest, the initial condition for the controller coordinate is then $x_{c}=\dot{x}_{c}=0$. For endpoint positioning control, however, the initial conditions for the controller coordinate must be chosen such that in the desired final configuration of the manipulator, the virtual spring and dashpot are in their equilibrium and unstretched position. In the end-point control problem, the induced vibrations are also suppressed by this controller design.

Remark 5: When the controllers are interpreted in physical terms, recall that for the case of displacement feedback, the position vector of the controller (virtual) mass $x_{c}$ in the Liapunov function, Eq. (22), is defined with respect to an inertial reference. However, for displacement and acceleration feedback, Eq. (30), it is defined relative to the actuator location. When the control mass location is expressed as an inertial position, the Liapunov function in the second case becomes identical to that in the first case. The difference is in the formulation of the control law. By expressing it in terms of the relative position of the controller mass, the scheme can be implemented by acceleration measurements without the need for velocity measurements.

\section{NUMERICAL EXAMPLES}

The robot system, shown in Fig. 9, consists of six one degrec-of-freedom joints, namely, shoulder pitch, yaw, elbow pitch, wrist yaw, pitch and roll. This robot model has the same kinematic relationships and mass properties as the Shuttle's Remote Manipulator System (RMS). The shoulder and elbow joints are connected by a 6.4 meter long lightweight carbon composite boom. This is designated the upper arm boom. The lower arm boom, connecting the elbow joint to the wrist joints, is approximately 7 meters long. The shoulder and elbow joints provide three translational degrees of freedom, and the wrist joints provide three additional rotational degrees of freedom of the end effector. Mass properties of each of the components of the robot system are given in Table 1.

A simple maneuver to move the end effector from its current position to a new position is simulated. Initially, the robot system is in the position shown in Fig. 9, and the position vector of the end effector is (15.3162, $0.3048,0.0$ ) given in terms of its $x, y, z$ components. The desired new position of the end effector relative to the robot base is $(10.3162,5.3048,5.0)$. Assume that all the wrist joints are locked during the maneuvering, and control torques arc only applied to each movable joint, i.e., the shoulder yaw, pitch, and elbow pitch joints. Since the system is driven by torques at the joints, the required change in each joint angle is pre-calculated to be $-0.45131 \mathrm{rad},-0.3111 \mathrm{rad}$, and $1.2106 \mathrm{rad}$ for the shoulder yaw, pitch, and elbow pitch joints, respectively. In this case, a second-order virtual rotational spring-mass damper system is located at each joint to be controlled. The controller system then has virtual moments of inertia 
instcad of virtual masses, torsional springs and dashpots instcad of lincar springs and dashpots. The initial conditions of the control system arc chosen such that in the final desired configuration of the manipulator, the virtual springs assume their free-length forms.

In the simulations, the wrist links are modeled as rigid bodies since they are fairly short relative to the lengths of both arms. The upper and lower arms are modeled as flexible bodies. The geometric and material properties of the flexible arms are given in Table 2. Only the first three cantilever modes are used to represent the flexibility of the links. Three different controllers, distinguished by the measured signals, are used to illustrate the use of the controller design methods in this paper.

\section{Case 1: Displacement and velocity feedback}

Case 1 uses angular displacements and velocities of joints for feedback. The controllers for each joint are rotational versions of the diagram in Fig. 6 with the bottom connection associated with acceleration removed. The parameters of the controller are given in Table 3. No attempt has been made to optimize the parameters chosen. Figures 10-12 show time histories of the end effector locations in both flexible and rigid-body simulations. The results show that the vibration is actively suppressed. Figure 13 shows the time history of the dominant first mode of the lower arm during this maneuver.

\section{Case 2: Displacement feedback}

One advantage of the virtual system controller design approach developed here is that velocity feedback is not required for vibration control or stability robustness. The stability robustness however, need nol guarantee good damping rates. To attempt to produce responses like those in Case 1, but this time using displacement measurement fecdback alone, the order of the controller is increased to cmulate velocity feedback. This is done by replacing the velocity feedback portion of Case 1 with a rotational virtual system corresponding to Fig. 2, and using a large $k_{c_{1}}$ and a small $m_{c}$. The control structure can again be vicwed by comparing to Fig. 6 . The bottom acceleration feedback connection is removed as before, and in place of the middle velocity conncction, we substitute a repeat of the top connection, but with a different choice of parameters. Theoretically, when both the $k_{c}$ and $m_{c}$ of the middle connection go to their limits, the control system in Case 2 is equivalent to the control system in Case 1. Numerically, the values of these constants are constrained by the introduction of high frequencies and real-time numerical integration difficulties.

In the simulation, the value of $k_{q}$ of the second virtual system is taken to be 100 times stiffer than the $k_{c_{1}}$ of the first virtual system. Figure 14 shows the simulation results with two different values of $m_{c}$ for the second virtual system, and shows that this approach will require rather extreme mass and stiffness values for good damping performance.

\section{Case 3: Displacement and displacement-acceleration} feedback

Another way to emulate the velocity signal is by using displacement-acceleration feedback. By replacing the velocity feedback in Case 1 with the displacementacceleration feedback of Fig. 4 and using large $k_{c_{2}}$ and small $m_{c}$ in this virtual system, the controller works similarly to the controller used in Case 1. It can be viewed as a rotational version of Fig. 6 with the center velocity connection removed. Theoretically, when the $k_{c_{2}}$ and $\boldsymbol{m}_{\boldsymbol{c}}$ of the acceleration connection go to their limits, the control system in Case 3 is equivalent to the control system in Case 1 . The controller in Case 3 is numerically better conditioned than that in Case 2 , since the velocity signal is obtained in the limit through integration of acceleration rather than differentiation of displacement.

In the simulation, the value of $k_{c_{2}}$ of the second virtual system is taken to be 100 times stiffer than the $k_{c_{2}}$ of the first virtual system. The results obtained are identical to within plotting accuracy, to the results obtained in Case 1 , and are therefore not shown here. Since acceleration fecdback is often more realistic than velocity feedback, this example shows that the control design of Case 3 can be important in practical applications.

So far, the controller parameters have not been optimized. To get some understanding of how the parameters of the virtual system affect performance, several cases are

simulated with adjusted $m_{c}, k_{c_{2}}$, and $d_{c}$ of the first virtual control system in Case 3. Each of the parameters is changed to ten times larger and ten times smaller than its original value. Figure 15 shows the angular displacement of the elbow pitch joint, for different values of $k_{c_{2}}$. Faster response can be achieved by using a larger spring constant $k_{c_{2}}$, as the results revealed. Figure 16 gives results obtained when varying the control mass $m_{c}$. In this particular example, the smaller the controller mass, the better the response. Figure 17 presents the results of varying the control damping constant $d_{c}$. Note that the use of a large damping constant may result in slow system response.

\section{CONCLUSIONS}

In this paper, a general control design methodology has been outlined for large angle position control and vibration suppression in multiple flexible-body dynamic systems. The method guarantees stability of the 
controlled system, whether linear or non-linear. The approach is model-independent in the sense that knowledge of the system dynamics is not required in the design process. Hence, it is robust with respect to parameter variations. Unlike positive real controller design which allows velocity measurements only, the current development can use velocity, position, and acceleration measurements or any combination, and still guarantees stability of the closed loop system. In addition it allows use of dynamic feedback controllers with additional opportunity for tuning to obtain good system performance. One special case of the methodology demonstrates how one can obtain the desirable properties of velocity feedback using acceleration feedback, which is often easier to instrument in practice. The design has intuitive appeal in terms of its physical interpretation that aids the control design process.

\section{REFERENCES}

1 JUANG, J.-N. and PHAN, M., "Robust Controller Designs for Second-Order Dynamic Systems: A Virtual Passive Approach," NASA Technical Memorandum TM102666, May 1990.

$2 \mathrm{OH}$, H-S., VADALI. S.R., and JUNKINS, J.L., "Use of the Work-Energy Rate Principle for Designing Feedback Control Laws," Journal of Guidance, Control. and Dynamics, to appear.
3 CANAVIN, J.R., "The Control of Spacecraft Vibration Using Multivariable Output Feedback," Proceedings of the AIAA/AAS Astrodynamics Conference, Paper No. 781419, Palo Alto, California, August 1978.

4 POPOV, V.M., Hyperstability of Automatic Control Systems, Springer-Verlag, New York, 1973.

5 POPOV, V.M., Lectures on Siability Theory, University of Southem California, Electrical Engineering Technical Report 357, 1969.

6 NEWCOMB, R.W., Linear Multiport Synthesis, McGraw-Hill, New York, 1966.

7 BENHABIB R.J., IWENS, R.P., and JACKSON, R.L., "Stability of Large Space Structure Control Systems Using Positivity Concepts," Journal of Guidance, Control, and Dynamics, Vol. 4, No. 5, Sept.-Oct. 1981.

8 FLASHNER, H., "An Approach for Control of Robot Manipulators," Proceedings of the AIAA Guidance. Navigation, and Control Conference, Snowmass, Colorado, August 1985.

9 SEVASTON, G. and LONGMAN, R.W., "Optimal Positive Real Controllers for Large Space Structures," Proceedings of the Third VPI\&SUIAIAA Symposium on Dynamics and Control of Large Flexible Spacecraft, L. Meirovitch, Editor, Blacksburg, Virginia, June 1981. 
Table 1 Mass Properties of the Robot System

\begin{tabular}{|c|c|c|c|c|c|c|c|}
\hline Name & Mnss (kB) & $1 \times x\left(\mathrm{~kg}_{\mathrm{b}}-\mathrm{m}^{2}\right)$ & $\lg y\left(k_{f}-m^{2}\right)$ & I $z: 2\left(\mathrm{k}_{\mathrm{B}}-\mathrm{m}^{2}\right)$ & $\operatorname{lxy}\left(\mathrm{kg}_{\mathrm{B}}-\mathrm{m}^{2}\right)$ & $\mid x z \cdot\left(k g-m^{2}\right)$ & Tyz $\left(k_{B}-m^{2}\right)$ \\
\hline Base & 32.11 & 11.1416 & 5.95196 & 14.1681 & $0.7958 \times 10^{-}$ & $0.1925 \times 10^{-}$ & $.0 .366 \times 10^{-1}$ \\
\hline Lower Arm & 26.50141 & $0.1351 \times 10^{-1}$ & 97.43457 & 97.4181 & 0.2945 & 0.0 & 0.0 \\
\hline Upner Ann & 33.3819 & $0.2458 \times 10^{-1}$ & 126.9785 & 127.0031 & .0 .52954 & 0.0 & 0.0 \\
\hline Wrist (Picli) & 8.4675 & 8.921161 & 4.01316 & 1.0267 & 0.0 & 0.0 & 0.0 \\
\hline Wrist (Yniv) & 15.97 & A.0809 & 1.67751 & 1.68022 & $-0.7 .321 \times 10^{-2}$ & $0.1164 \times 10^{-2}$ & $-0.2928 \times 10^{-3}$ \\
\hline Wrisi (Roll) & 75.21 & T.90נ54 & 2.8277 & 3.81657 & 0.0 & 0.0 & 0.0 \\
\hline Shoniter Juini & Tok.MI & $\pi .0$ & 0.0 & 0.0 & 0.0 & 0.0 & 0.0 \\
\hline (tilx)w Jimili & 61.00 & 0.0 & 0.0 & 0.0 & 0.0 & 0.0 & 0.0 \\
\hline
\end{tabular}

Tnble 2 Gcometrics of the Robot System and Material

Properties of Flexible Links

\begin{tabular}{|c|c|c|c|c|c|c|c|}
\hline Nnina & Longll $(n)$ & I: $\left(\mathrm{N} / \mathrm{m}^{2}\right)$ & C $\left(\mathrm{N} / \mathrm{m}^{2}\right)$ & $A\left(m^{2}\right)$ & $p\left(\mathrm{~kg}_{\mathrm{g}} / \mathrm{m}_{1}{ }^{3}\right)$ & $11=12\left(\mathrm{~m}^{4}\right.$ & $J\left(m^{4}\right)$ \\
\hline$U_{p p e r} \lambda_{r m:}$ & $6.375 \mathrm{~A}$ & & & & & & \\
\hline Section 1 & 0.7239 & $1.20 \times 10^{\top 1}$ & $4.615 \times 10^{10}$ & $1.3687 \times 10^{-3}$ & 4540.0 & $1.8683 \times 10^{-3}$ & $2.3313 \times 10^{-5}$ \\
\hline Scction 2 & 5.0038 & $1.58 \times 10^{11}$ & $3.98 .5 \times 10^{10}$ & $1.9662 \times 10^{-\mathrm{J}}$ & 2504.8 & $2.6605 \times 10^{-5}$ & $5.3206 \times 10^{-5}$ \\
\hline Scclion 3 & 0.3302 & $1.20 \times 10^{11}$ & $4.615 \times 10^{10}$ & $1.3687 \times 10^{-J}$ & 1510.0 & $1.1886 \times 10^{-5}$ & $1.6177 \times 10^{-5}$ \\
\hline Seclion 4 & 0.3522 & $1.20 \times 10^{11}$ & $4.615 \times 10^{10}$ & $1.3687 \times 10^{-J}$ & 15.10 .0 & $1.1886 \times 10^{-5}$ & $1.6477 \times 10^{-5}$ \\
\hline Lower Arm: & 7.0612 & & & & & & \\
\hline Seclion 1 & 0.3522 & $1.20 \times 10 \mathrm{~T}$ & $4.615 \times 10^{10}$ & $7.411 \times 10^{-1}$ & 1540.0 & $1.0080 \times 10^{-5}$ & $1.7410 \times 10^{-3}$ \\
\hline Section 2 & 5.7404 & $1.55 \times 10^{11}$ & $4.07 \mathrm{~A} \times 10^{10}$ & $1.308 \times 10^{-5}$ & 3161.7 & $1.8189 \times 10^{-5}$ & $3.63 \times 2 \times 10^{-5}$ \\
\hline Section 3 & 1.0033 & $1.20 \times 10^{11}$ & $4.615 \times 10^{10}$ & $3.390 \times 10^{-1}$ & 4540.0 & $1.5920 \times 10^{-6}$ & $6.4050 \times 10^{-6}$ \\
\hline Bnsc & $0.30 \mathrm{M}$ & & & & & & \\
\hline TWrist (Pich) & 0.1572 & & & & & & \\
\hline Wrist $(Y n w)$ & 0.7620 & & & & & & \\
\hline Wrisi (Rolli) & $0.661 \mathrm{M}$ & & & & & & \\
\hline
\end{tabular}

Table 3 Parnmeters of Virtual Systems for the Robot Maneuvering Control willı Velocity and Displacement Feedback

\begin{tabular}{|l|c|c|c|c|c|}
\hline Joint Name & $m_{c}$ & $k_{c l}$ & $k_{c 1+k_{c 2}}$ & $J_{c}$ & $d$ \\
\hline Shoulder Yaw & $0.25639 \times 10^{5}$ & 1011169.0 & 2000000.00 & 225419.0 & 274581.00 \\
\hline Shoulder Pitcl & $0.25639 \times 10^{5}$ & 1011169.0 & 2000000.00 & 225419.0 & 274581.00 \\
\hline E.l6ow Piich & $0.65157 \times 10^{-1}$ & 256970.00 & 500000.00 & 57286.00 & 42714.00 \\
\hline
\end{tabular}




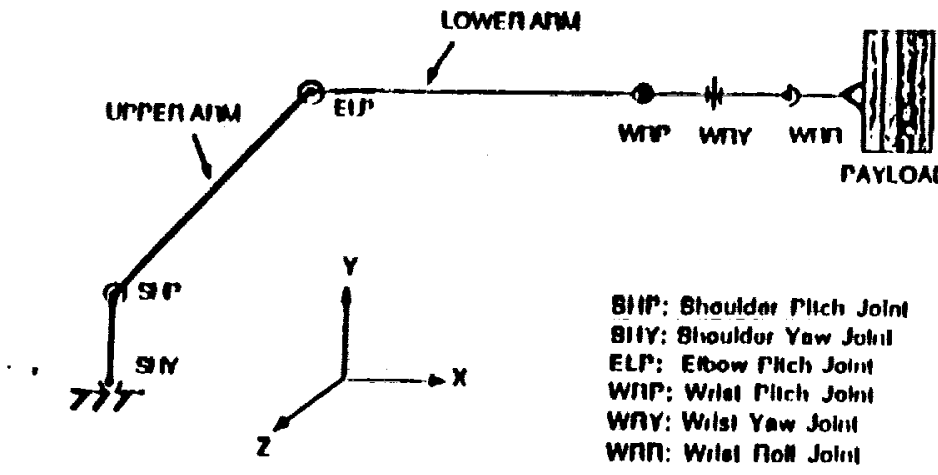

Figure 9: $\wedge$ Six-Degree-of-Freedon Robot

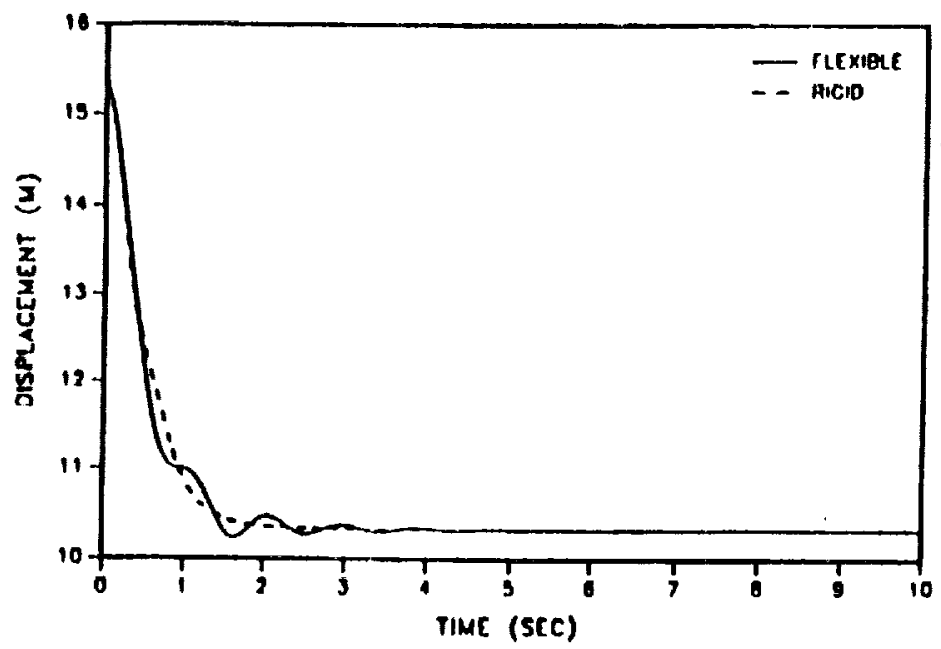

Figure 10: Time Ilistories of the $\mathrm{x}$-Position of the End Effector, Case 1

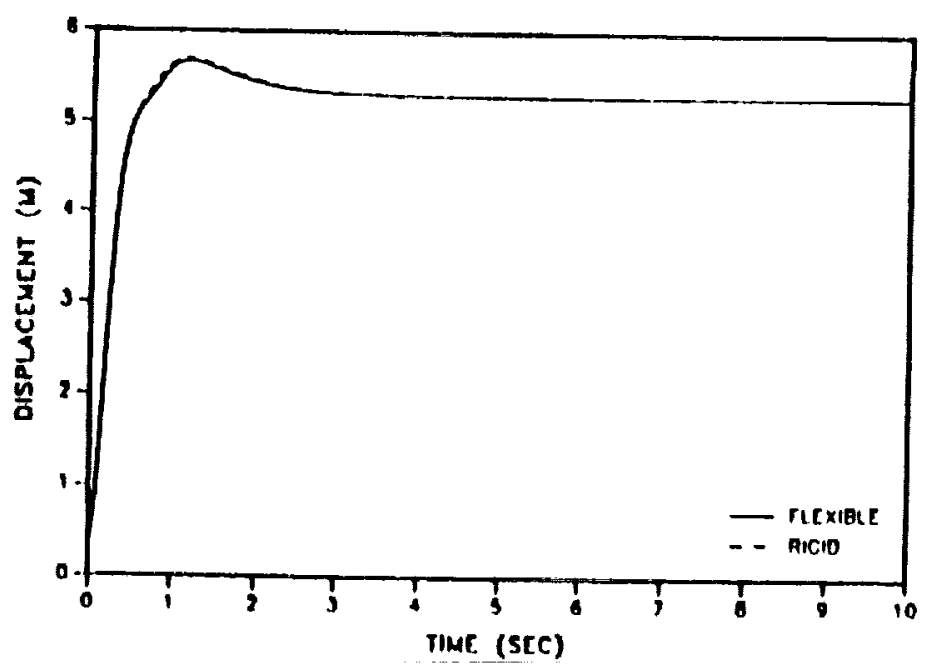

Figure 11: Time Ilistories of the $y$-Position of the End Effector, Case 1 


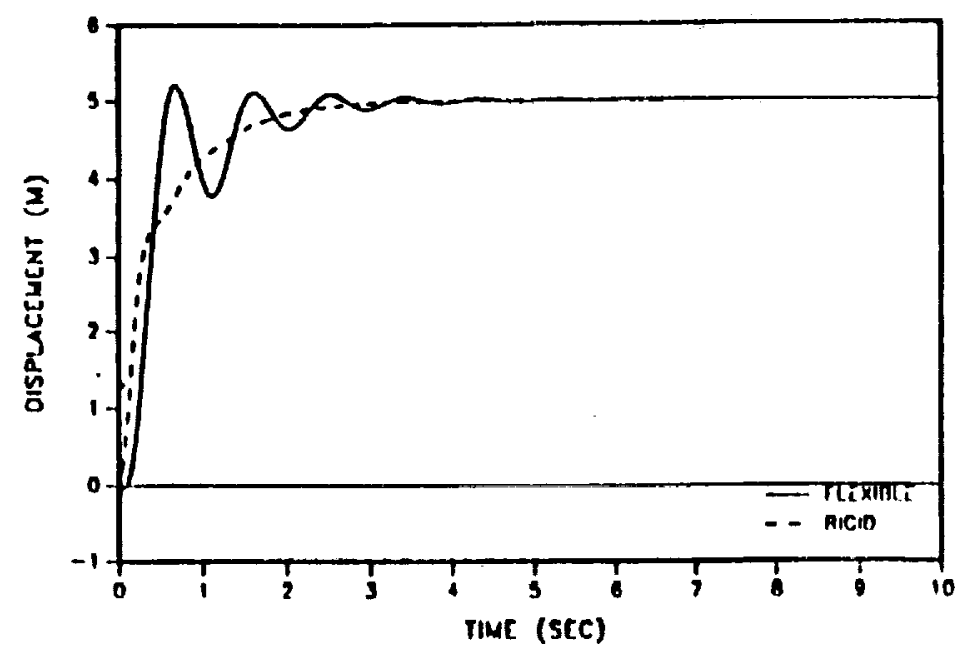

Figure 12: Tine Ilistories of the 2-Position of the End Erfector, Cuse 1

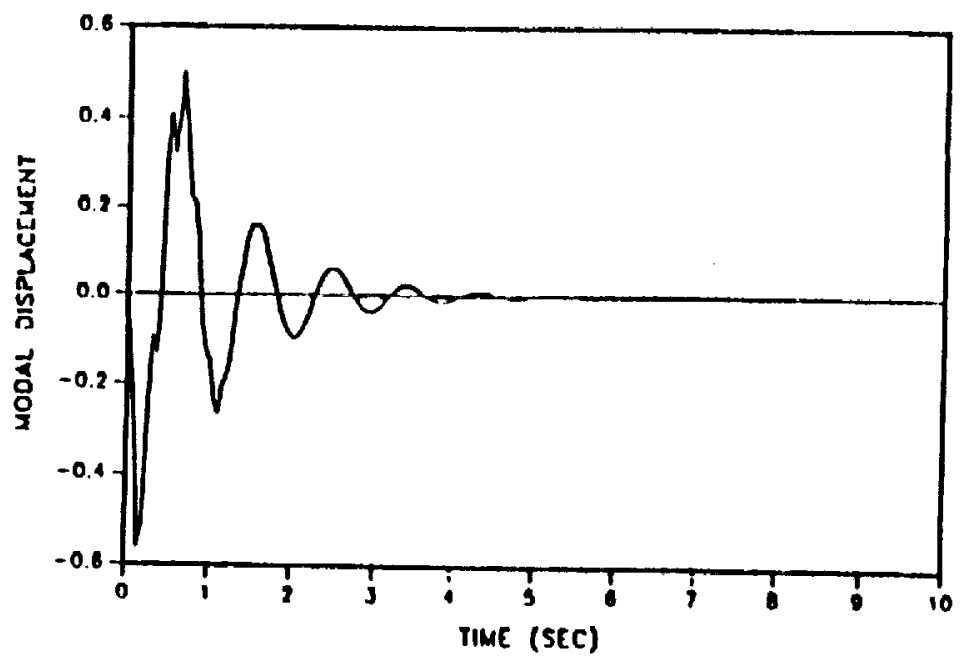

Figure 13: Time llistories of the First Mode of the Lower Arm during Maneuver

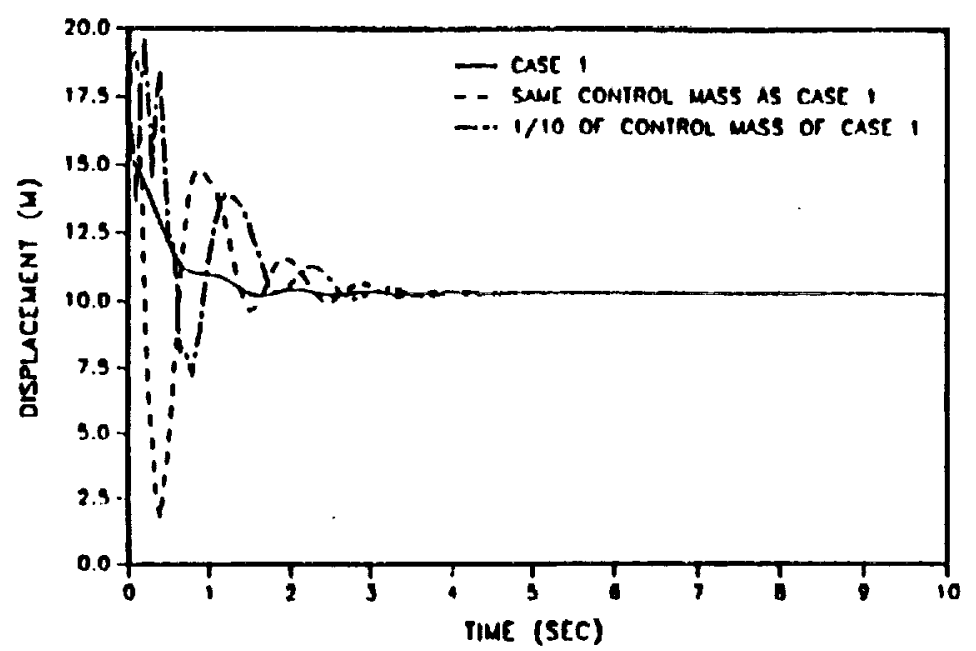

Figure 11: Tine Ilistories of the $x$-Position of the End Efrector, Case 2 


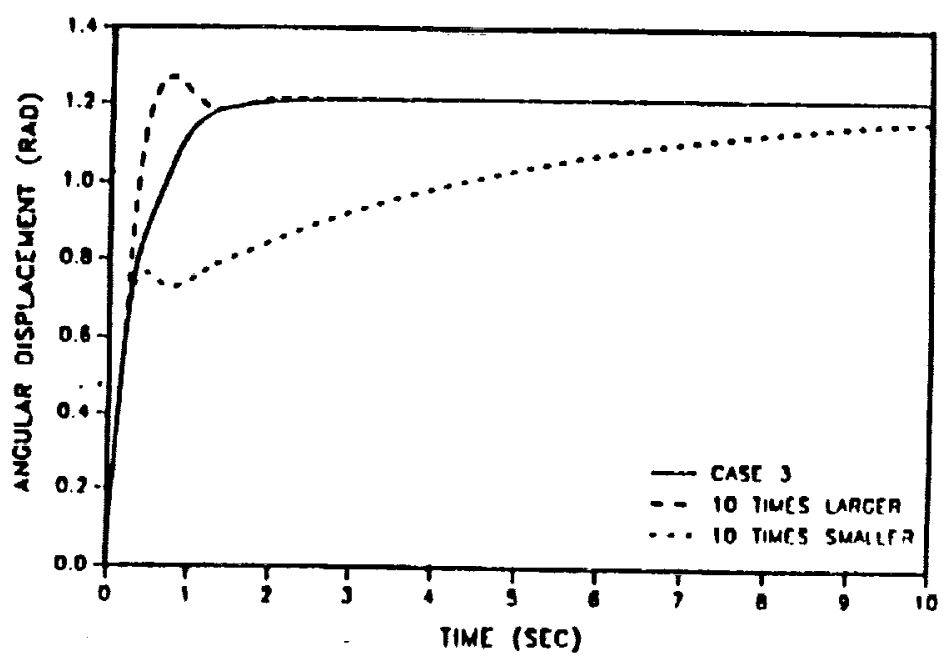

Figure 15: Time IJistories of the Displncement of the Eilbow Pitch for Different Vulues of Control Stifrness Constant

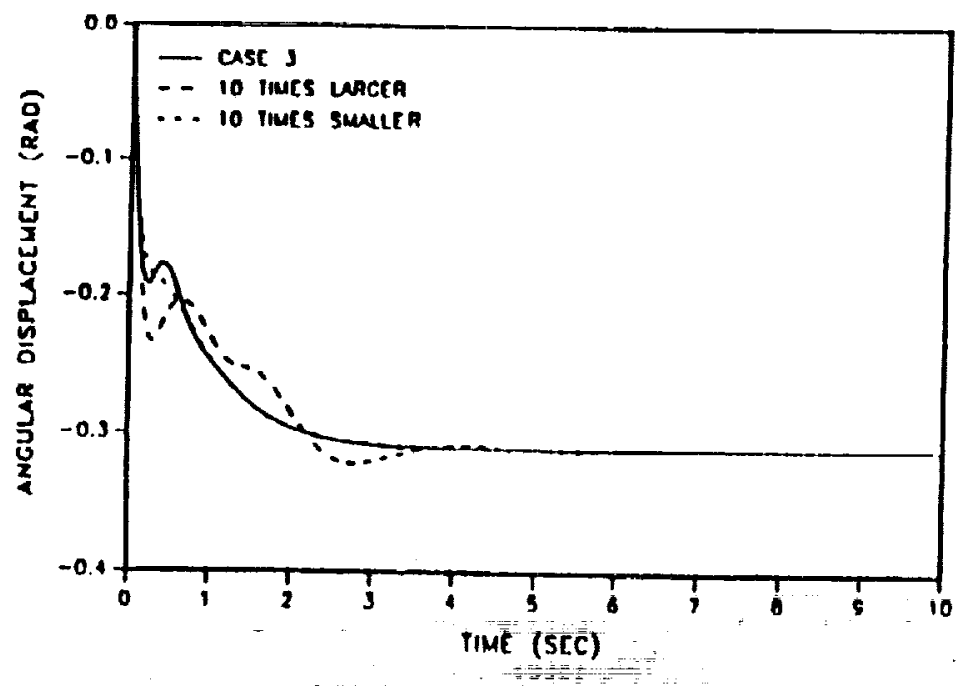

Figure 16: 'Time Ilistories of the Displacenent of the Shoulder Pitch for Different Values of Control Mnss Constant

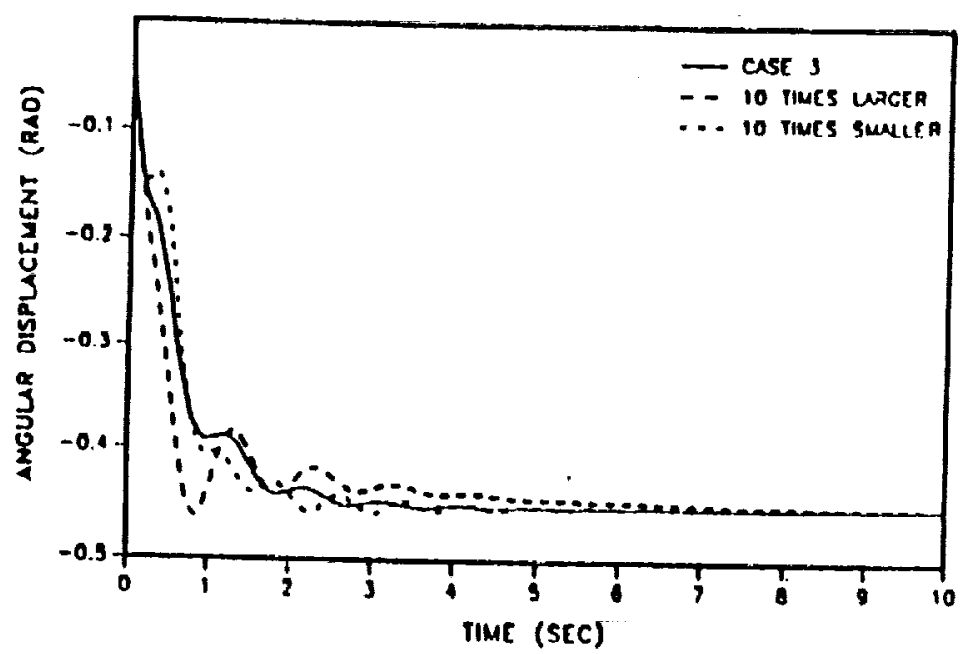

Iigure 17: Time Ilistories of the Displncement of the Shoulder Yaw for Different Vulues of Control Dunuping Constant 
NAS 7 TM-104047

\section{Title and Subtitle}

Passive Dynamic Controllers for Non-Linear Mechanical Systems

\section{Report Date \\ March 1991}

6. Pertorming Organization Code

\section{Author(s)}

Jer-Nan Juang, Shih-Chin Wu, Minh Phan, and Richard W. Longman

\section{Performing Organization Name and Address \\ NASA Langley Research Center \\ Hampton, VA 23665-5225}

\section{Sponsoring Agency Name and Address}

National Aeronautics and Space Administration washington, DC 20546-0001

\section{Performing Organization Peport No.}

10. Work Unit No.

590-14-21-01

11. Contract or Grant No.

13. Type of Report and Period Covered

Technical Memorandum

14. Sponsoring Agency Code

\section{Supplementary Notes}

Presented at the 32 nd Structures, Structural Dynamics and Materials Conference Baltimore, MD, April 8-10, 1991.

Jer-Nan Juang, Langley Research Center, Hampton, Virginia; Shih-Chin Wu, Lockheed Engineering \& Sciences Company, Hampton, Virginia; Minh Phan and Richard w. Longman, National Research Council Research Associates, Langley Research Center, Hampton, VA.

\section{Abstract}

A methodology for model-independent controller design for controlling large angular motion of multi-body dynamic systems is outlined. The controlled system may consist of rigid and flexible components that undergo large rigid body motion and small elastic deformations. Control forces/torques are applied to drive the system, and at the same time suppress the vibration due to flexibility of the components. The proposed controller consists of passive second-order systems which may be designed with little knowledge of the system paramenter, even if the controlled system is non-linear. Under rather general assumptions, the passive design assures that the closed loop system has guaranteed stability properties. Unlike positive real controller design, stabilization can be accomplished without direct velocity feedback. In addition, the second-order passive design allows dynamic feedback controllers with considerable freedom to tune for desired system response, and to avoid actuator saturation. After developing the basic mathematical formulation of the design methodology, simulation results are presented to illustrate the proposed approach to a flexible six-degree-of-freedom manupulator.

\section{Key Words (Suggested by Author(s))}

Nonlinear Dynamics and Control

Multibody Dynamics and Control

Model-Independent Controller Design

Dissipative Controller Design

\section{Distribution Statement}

Unclassified--Unlimited

Subject Category 39
19 Security Classif. (of this report)

Unolasis is icd
20. Security Classif. (ot this page) Unclassified
21. No. of pages

15
22. Price

$\mathrm{AO} 3$ 
